\title{
Plasma Fibrinogen to Platelet Ratio as a Prognostic Factor for Postoperative Recurrence of Esophageal Squamous Cell Carcinoma
}

Jinye Zhang ( $\sim$ JYKzjy@126.com )

Nantong daxue fushu zhongliu yiyuan: Nantong Tumor Hospital https://orcid.org/0000-0002-30530438

\section{Mingxin Shi}

Nantong daxue fushu zhongliu yiyuan: Nantong Tumor Hospital

\section{Liyuan Guo}

Nantong University

\section{Qiuxing Yang}

Nantong daxue fushu zhongliu yiyuan: Nantong Tumor Hospital

\section{Research}

Keywords: esophageal squamous cell carcinoma recurrence fibrinogen/platelet ratio prognosis

Posted Date: October 1st, 2021

DOI: https://doi.org/10.21203/rs.3.rs-870794/v1

License: (c) (i) This work is licensed under a Creative Commons Attribution 4.0 International License. Read Full License 


\section{Abstract}

Background: Postoperative recurrence of esophageal squamous cell carcinoma is an important factor affecting the survival of patients. Hypercoagulability is a related factor for the recurrence of esophageal squamous cell carcinoma. Fibrinogen/platelet ratio (FPR) is used as a meaningful factor for evaluating coagulation status. The purpose of this study is to evaluate the predictive value of FPR for ESCC postoperative metastasis and its relationship with prognosis.

Methods: We collected 607 patients undergoing surgery from 2012 to 2015, and performed clinical analysis, including plasma fibrinogen count, platelet count, and their ratio, defined as FPR. Follow-up assessment of recurrence and OS, RFS.

Results: 145 patients (23.8\%) experienced postoperative recurrence. There is a correlation between FPR and postoperative metastasis ( $\mathrm{p}<0.001)$. The area under the curve (AUC) of FPR predicting postoperative recurrence is 0.620 (95\% confidence interval: 0.554-0.686), which is higher than the single factor AUC value of fibrinogen and platelets. The overall survival rate (OS) and recurrence-free survival rate (RFS) of patients with higher FPR were significantly reduced $(p=0.001)$. Cox regression results show that FPR is an independent predictor of OS and RFS ( $p=0.004$ and $<0.028$, respectively), while plasma fibrinogen level is not. The $C$ index of the nomogram model predicting OS and RFS is 0.691 and 0.727 , respectively. The calibration curve shows that the predictive ability is basically consistent with the actual observation results.

Conclusions: FPR may be a predictor of postoperative recurrence in ESCC patients, and it can predict the prognosis of ESCC patients better than fibrinogen.

\section{Introduction}

Esophageal cancer is one of the most common malignant tumors in the world, and its morbidity and mortality rank seventh and sixth among all tumors ${ }^{[1]}$. Esophageal cancer can be divided into esophageal squamous cell carcinoma (esophageal squamous cell carcinoma, ESCC) and esophageal adenocarcinoma (EAC). In my country, ESCC is more common, accounting for about $95 \%$ of esophageal cancer cases. Although the treatment methods of esophageal cancer continue to improve, according to the data of the National Cancer Center, the five-year survival rate of esophageal cancer is only $30 \%$. Postoperative recurrence is the main reason for the poor prognosis. Therefore, it is necessary to further search for effective tumor serum markers. To improve the prognosis. Postoperative metastasis of ESCC occurs most often in lymph nodes, followed by lung, liver, and bone ${ }^{[2]}$. There are many factors that cause tumor metastasis, including hypercoagulable state and inflammation. There is evidence that inflammation and the coagulation system interact to promote tumor progression ${ }^{[3]}$. Hyperferricemia is also associated with the poor prognosis of a variety of solid tumors, and compared with non-relapsed cases, relapsed cases have higher fibrinogen ${ }^{[4]}$.Thrombocytosis reflects tumor-related inflammation. An increase in platelets ${ }^{[5]}$ and an increase in fibrinogen indicate a poor prognosis for $\operatorname{ESCC}^{[6-7]}$. 
Hyperfibrinogenemia is closely related to postoperative metastasis of ESCC ${ }^{[8]}$. In recent years, serum markers for predicting postoperative tumor recurrence have attracted much attention. However, so far there are few applications and clinical applications. Recently, the relationship between hypercoagulability and inflammation and tumor metastasis has attracted much attention. We speculate that the combination of fibrinogen and platelets can predict postoperative recurrence and prognosis of ESCC better than fibrinogen or platelets. Therefore, in this study, we collected 607 cases of clinical factors that have predictive significance for postoperative metastasis, and evaluated the predictive value of FPR for postoperative recurrence of ESCC and its impact on overall survival and recurrence-free survival.

\section{Materials And Methods}

\section{patients}

A selection of 607 patients with esophageal squamous cell carcinoma diagnosed pathologically from January 2012 to December 2015 in the Affiliated Tumor Hospital of Nantong University. Inclusion criteria: pathologically diagnosed as squamous cell carcinoma and undergoing surgical treatment; no radiotherapy and chemotherapy before surgery; no history of other malignant tumors. Exclusion criteria: preoperative history of infection, liver disease, blood disease; lack of clinical data; unwilling to follow-up and lost to follow-up patients. This study was approved by the Ethics Committee of the Affiliated Tumor Hospital of Nantong University. The clinical data included in this study include age, gender, smoking history, drinking history, tumor size, histological grade, tumor differentiation, lymph node status, pTNM stage, nerve invasion, vascular infiltration, adjuvant therapy and laboratory test results, including fibrinogen, platelets. According to the 2020 Chinese Society of Clinical Oncology (CSCO) Guidelines for Diagnosis and Treatment of Esophageal Cancer, the stage of the disease is determined. According to immunohistochemistry, determine the degree of tumor differentiation and histological grade.

\section{Collection Of Specimens}

Approximately $2 \mathrm{ml}$ of venous blood was drawn the morning before the operation when the patient was not eating for the detection of fibrinogen and platelets, FPR $=$ fibrinogen/platelets. According to the normal reference range of the cancer hospital, hyperfibrinogenemia is defined as: plasma fibrinogen level is greater than $4.0 \mathrm{~g} / \mathrm{L}$; thrombocytosis is defined as: platelet count greater than $300 \times 109 / \mathrm{L}$.

\section{Follow-up}

It will be reviewed every 3 months for the first two years after surgery, every 6 months for the next 3 years, and once a year thereafter. Follow-up methods include telephone follow-up and regular outpatient review (including clinical examination, imaging evaluation, serological examination). Recurrence is defined as clinical, imaging, or pathological diagnosis to find a previous local or distant tumor. OS is defined as the 
time from surgery to the patient's death from any cause or the last follow-up date when the patient is known to survive. RFS is calculated from the date of surgery to the first tumor recurrence or death.

\section{Statistical analysis}

Chi-square test was used to evaluate the relationship between clinicopathological variables and postoperative recurrence. The receiver operating characteristic (ROC) curve was used to determine the best cut-off values of fibrinogen, platelets, and FPR, and the predictive value of each variable was evaluated. Kaplan-Meier survival analysis was used to evaluate OS and RFS. Using Cox proportional hazards model, variables with $\mathrm{p}$ value $<0.05$ in univariate analysis were included in multivariate survival analysis to determine independent prognostic factors. Then select the nomogram for the independent prognostic factors in the COX regression model, and use the $\mathrm{R}$ language to calculate it to predict OS and RFS. The 3-year and 5-year prediction probability of the calibration chart is generated by comparing the nomogram, and the prediction accuracy is evaluated by 1000 times of guided resampling. All statistical analyses were performed using SPSS 26.0 software (SPSS, Inc. Chicago, Illinois, USA) and R statistical software. All data $p$-values $<0.05$ are considered statistically significant.

\section{Results}

\section{The relationship between clinicopathological parameters and postoperative metastasis}

A total of 607 patients were admitted, of which 145 patients experienced postoperative recurrence, and the remaining 462 patients were assigned to the non-recurrence group. Through ROC curve analysis, the best cut-off values of fibrinogen, platelets and FPR are 2.94g/L, 233*10[9]/L, 0.01553, respectively. Table 1 shows the relationship between clinicopathological parameters and postoperative recurrence of 607 patients. The results showed that there were significant differences in the common clinical characteristics of the two groups in age, tumor differentiation, histological grade, $\mathrm{N}$ stage, vascular infiltration, nerve invasion, radiotherapy and chemotherapy, fibrinogen level and FPR (all p values $<0.05$ ). There were no significant differences in gender, smoking history, drinking history, tumor location and size, T stage, $\mathrm{M}$ stage, and platelet level.

The predictive ability of fibrinogen, platelet and FPR for postoperative recurrence

Calculate the AUC values of fibrinogen, platelets and FPR, and the AUC values are $0.6070 .543-0.671$, $0.447(0.378-0.516), 0.620(0.554-0.686)$, respectively, indicating that FPR is better than a single fibrinogen and platelet count for ESCC surgery The prediction of recurrence is more meaningful (Table 2 and Fig. 1).

\section{Fpr And Prognosis}


The follow-up time for OS and RFS was 60 months. As shown in Fig. 2, compared with the low FPR group, the OS ( $p=0.001)$ and RFS $(p<0.001)$ of the higher FPR group were worse. Univariate Cox regression analysis (Table 2) showed that gender, histological grade, degree of tumor differentiation, tumor length, T stage, $\mathrm{N}$ stage, vascular invasion, nerve infiltration, postoperative recurrence, fibrinogen level, and FPR level are the factors that affect OS Prognostic factors with RFS. Multivariate Cox regression analysis showed that T stage $(p=0.001), N$ stage $(p<0.001)$, postoperative recurrence $(p<$ $0.001)$ and FPR $(p=0.004)$ are independent prognostic factors of OS; at the same time, T stage $(p<$ $0.001)$, $N$ stage $(p<0.001)$, postoperative recurrence $(p<0.001)$ and FPR $(p=0.028)$ are also independent prognostic factors of RFS.

\section{Prognostic Nomograms Of Os And Rfs}

In order to better predict the OS and RFS of ESCC patients, the meaningful single factor factors in the COX review model were selected to establish two nomograms, and the Harrell's $\mathrm{c}$ index was used to evaluate the prediction accuracy of the model. The prognostic nomogram of all important independent factors of comprehensive multivariate analysis is shown in Fig. 3. The $c$ index of OS is 0.691 , and the $C$ index of RFS is 0.727 . As shown in Fig. 4 , the calibration curve of the overall survival rate at 3 and 5 years after surgery has a fair fit, and the calibration curve for the recurrence-free survival rate at 3 and 5 years after surgery has a good fit. The results show that the FPR-based nomogram has a better predictive significance for OS and RFS, and the prediction of RFS has a better predictive significance for OS.

\section{Discuss}

Esophageal cancer is the sixth most deadly tumor in the world. Although the current treatment methods are constantly improving, the 5-year survival rate of patients is still poor, and postoperative recurrence is the most important factor for the poor prognosis of patients. Therefore, this study aims to explore whether FPR can early predict postoperative recurrence in patients and its prognostic significance for OS and RFS. FPR is the ratio of fibrinogen to platelets, which is more convenient to calculate and can be used to predict the prognosis of gastric cancer. In this study, we combined fibrinogen and platelets, taking the ratio, to explore the predictive significance of postoperative ESCC recurrence, as well as the predictive significance of OS and RFS. We have shown through research that FPR is a good predictor of postoperative ESCC recurrence. Previous studies have shown that fibrinogen has predictive significance for postoperative ESCC metastasis. On this basis, we have studied the predictive value of FPR for ESCC postoperative recurrence. Through our data analysis, FPR has a greater predictive value for postoperative recurrence than any single factor of fibrinogen and platelets. And it shows that FPR is an independent prognostic factor of OS and RFS, while fibrinogen and platelets are not. This study also established a nomogram to predict the disease-free survival of 3, 5 years and 3,5 years of overall survival after radical esophagectomy for esophageal cancer, and it can be seen that the calibration curve and the simulation curve fit well. 
There has been no research on FPR in esophageal squamous cell carcinoma. We know a lot of research on platelets and fibrinogen in tumor metastasis. Tumor metastasis is a multi-system regulation process, in which the blood vasculature plays an important role. Tumor cells fall off from the original site, enter the whole body through the circulatory system, and eventually colonize the target organs, resulting in tumor metastasis. Circulating tumor cells adhere to platelets, leukocytes and endothelial cells to make tumor cells exudate from the vasculature and promote tumor cells to colonize and survive far away ${ }^{[9]}$. Fibrinogen and platelets are linked to each other and jointly promote the development of tumors. Fibrinogen can promote the adhesion of platelets to tumor cells, and platelets form thrombin so that fibrinogen accumulates around tumor cells, thereby protecting tumor cells from natural killer cytotoxicity [10]. What mechanism does fibrinogen mainly promote tumor progression and metastasis? A lot of research is needed in the future. At present, some studies claim that tumors may be the source of fibrinogen. Fibrinogen, fibrin and its degradation products have Pro-inflammatory activity, they indirectly stimulate the endothelium to secrete von Willebrand factor, leading to platelet activation associated with neoplastic diseases ${ }^{[11]}$. Fibrinogen is converted into fibrin, and fibrinogen also promotes the formation of new blood vessels, so it can promote tumor growth and metastasis ${ }^{[12]}$. Fibrinogen levels are elevated in malignant tumors such as gastric cancer, esophageal cancer, breast cancer, lung cancer, colon cancer and ovarian cancer, and high fibrinogen indicates a poor prognosis. In gastric cancer, fibrinogen is associated with disease progression and recurrence ${ }^{[13]}$. In esophageal cancer, it has been reported that fibrinogen is related to lymph node metastasis and is a biomarker for predicting tumor progression, recurrence and prognosis of esophageal cancer ${ }^{[14]}$. Thrombocytosis is also closely related to tumor progression. Platelets promote blood clotting associated with cancer. Platelets are recruited to cover the surface of tumor cells, thereby protecting them from immune cell responses and promoting cancer growth and metastasis ${ }^{[15]}$. There are also reports that cancer cells migrate to the vasculature and interact with platelets, leading to platelet aggregation induced by tumor cells ${ }^{[16]}$. This shows that tumor cells and platelets have a mutually promoting relationship. Fibrinogen and platelets are elevated in a variety of cancers, such as lung cancer, cervical cancer, stomach cancer, colon cancer, pancreatic cancer, and prostate cancer. In pancreatic cancer, fibrinogen and platelets can predict tumor metastasis ${ }^{[17]}$.

Our research shows that FPR's AUC (0.620) is better than the other two indicators, but the AUC value is less than 0.8 , which is not high enough, and more data in the future are needed for the next step of verification. The limitations of this study include that this is a retrospective study, the data comes from a single organization, the sample size of the data is small, and the sample size needs to be increased to verify the accuracy of the FPR cutoff value and its impact on the prognosis. Secondly, the risk model we evaluated also comes from a single institution, and its applicability is relatively limited, and further multiinstitution research is needed. In summary, preoperative FPR is convenient, cheap, and simple to calculate. It can predict postoperative recurrence of ESCC patients better than plasma fibrinogen levels. Patients with high preoperative FPR are more likely to relapse after surgery, and have poor OS and RFS.

Potential limitations of this study include the use of a retrospective design. In order to select a more uniform patient background, we only included patients with esophageal cancer who were likely to be 
cured by surgery, and excluded patients with esophageal cancer who received neoadjuvant therapy, which may also limit the general application of the results of the study. In addition, larger-scale prospective studies are needed to confirm these preliminary results.

\section{Abbreviations}

FPR fibrinogen-to-platelet ratio

ESCC esophageal squamous cell carcinoma

OS overall survival

RFS recurrence-free survival

EAC esophageal adenocarcinoma

AUC Area under the curve

Cl Confidence interval

C-index Concordance index

ROC Receiver-operating characteristic

\section{Declarations}

\section{Authors' contributions}

GLY was involved in data analysis, interpretation and manuscript writing. GLY and YQX were involved in collection data. ZJY and SMX was involved in conception and design. All authors contributed to the article and approved the final manuscript.

\section{Funding}

The authors received no specific funding for this study.

\section{Availability of data and materials}

The datasets in this study is available by request from the corresponding

author. For more information, please contact the corresponding author.

\section{Ethics approval and consent to participate}

All the procedures followed were in accordance with the ethical guidelines 
of the Helsinki Declaration. The study protocol was approved by the Medical Ethical Committee of in the Affiliated Tumor Hospital of Nantong University. All patients were given informed written consent. And We received written consent from all patients.

\section{Consent for publication}

Not applicable.

\section{Competing interests}

The authors declare that they have no competing interests.

\section{Contributor Information}

Jinye Zhang, Email: JYKzjy@126.com

Mingxin Shi, Email:1017168199@qq.com

Liyuan Guo, Email:1553677009@qq.com

Qiuxing Yang, Email:2268552452@qq.com

\section{References}

1. Bray Freddie F, Jacques S, Isabelle, et al. Global cancer statistics 2018: GLOBOCAN estimates of incidence and mortality worldwide for 36 cancers in 185 countries.[J]. CA Cancer J Clin. 2018;68:394-424.

2. Quint LE, Hepburn LM, Francis IR, Whyte RI, Orringer MB, et al. Incidence and distribution of distant metas-tases from newly diagnosed esophageal carcinoma. Cancer. 1995;76:1120-5.

3. Cantrell Rachel, Palumbo Joseph S,et al. The thrombin-inflammation axis in cancer progression. [J].Thromb Res, 2020, null: S117-S122.

4. Perisanidis C, Psyrri A, Cohen EE. et al. Prognostic role of pretreatment plasma fibrinogen in patients with solid tumors: A systematic review and meta-analysis.[. J]Cancer Treat Rev. 2015;41:960 - 70.

5. Song Qian Wu, Sheng J-ZW. et al. Elevated preoperative platelet distribution width predicts poor prognosis in Esophageal. Squamous Cell Carcinoma[J]Sci Rep. 2019;9:15234.

6. Wang Jianbo L, Na HS. et al. The clinical significance of preoperative plasma fibrinogen level and platelet count in resectable esophageal squamous cell carcinoma.[J]. World J Surg Oncol. 2015;13:157.

7. Qiao Yufeng M, Mingquan, Zhang, Hongdian, et al. Prognostic Significance of the Combination of Fibrinogen and Tumor Marker Index in Esophageal Squamous. Cell Carcinoma Patients[J]Onco Targets Ther. 2021;14:1101-11. 
8. Zhang D,Zhou Xia,Bao Wuan, et al. Plasma fibrinogen levels are correlated with postoperative distant metastasis and prognosis in esophageal squamous cell carcinoma.[J]. Oncotarget, 2015, 6: 3841020.

9. Konstantopoulos Konstantinos, Thomas Susan N,et al. Cancer cells in transit: the vascular interactions of tumor cells.[J]. Annu Rev Biomed Eng. 2009;11:177-202..12.Interaction of fibrin with VE-cadherin.

10. Sheng Z, Jian S, Yang J, et al. Platelets and fibrinogen facilitate each other in protecting tumor cells from natural killer cytotoxicity.[. J]Cancer Sci. 2009;100:859-65.

11. Kołodziejczyk J,Ponczek Michał B,et al.The role of fibrinogen, fibrin and fibrin(ogen) degradation products (FDPs) in tumor progression.[J].Contemp Oncol (Pozn), 2013, 17: 113-9.

12. Martinez J, Ferber A, Bach TL, et al. Interaction of fibrin with VE-cadherin.[J]. Ann N Y Acad Sci. 2001;936:386-405.

13. Yamamoto Masaaki K, Yukinori M, Yasuhiro, et al. Usefulness of Preoperative Plasma Fibrinogen Versus Other Prognostic Markers for Predicting. Gastric Cancer Recurrence[J]World J Surg. 2016;40:1904-9.

14. Wakatsuki Kohei M, Sohei M, Kazuhiro, et al. Preoperative Plasma Fibrinogen is Associated with Lymph Node Metastasis and Predicts Prognosis in Resectable Esophageal Cancer.[J]. World J Surg. 2017;41:2068-77.

15. Bambace NM, Holmes CE,et al. The platelet contribution to cancer progression.[. J]J Thromb Haemost. 2011;9:237-49.

16. Jurasz Paul A-E, David R, Marek W, et al. Platelet-cancer interactions: mechanisms and pharmacology of tumour cell-induced platelet aggregation.[. J]Br J Pharmacol. 2004;143:819-26.

17. Wang Haiyan G, Jinbiao B, Ming, et al. The pretreatment platelet and plasma fibrinogen level correlate with tumor progression and metastasis in patients with pancreatic cancer.[. J]Platelets. 2014;25:382-7.

\section{Tables}

Due to technical limitations, tables 1 to 3 are only available as a download in the Supplemental Files section.

\section{Figures}


Figure 1

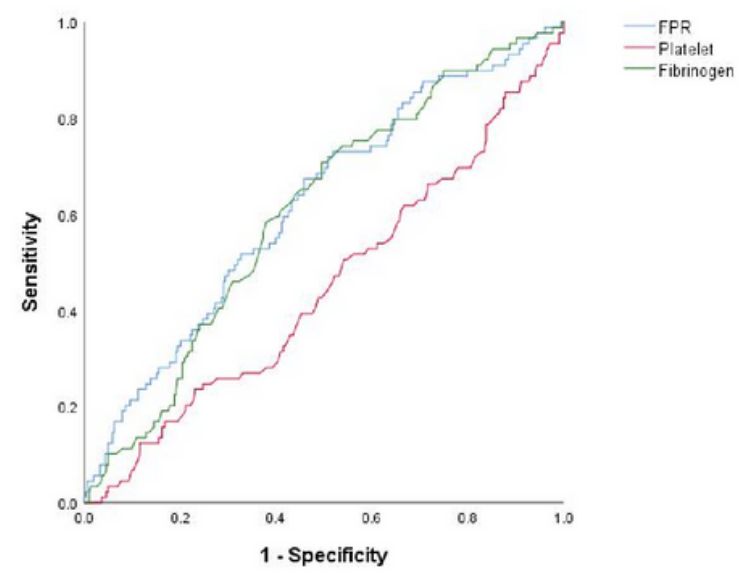

\section{Figure 1}

The receiver operating characteristic $(\mathrm{ROC})$ curves of Platelet, Fibrinogen and FPR for predicting recurrence. The area under the ROC curve (AUC) was 0.447 and 0.607 for Platelet, Fibrinogen, respectively. The AUC increased to 0.620 for the FPR.

Figure 2

a

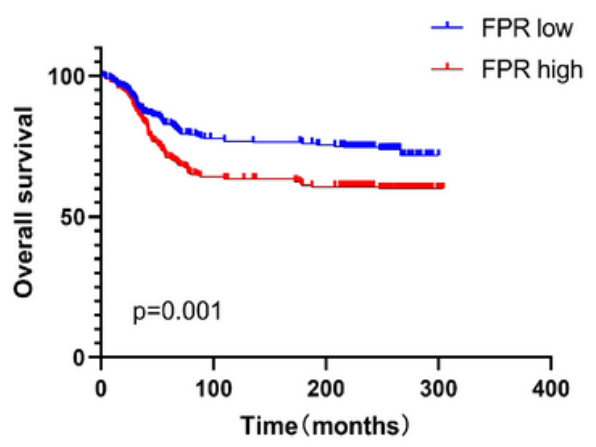

b

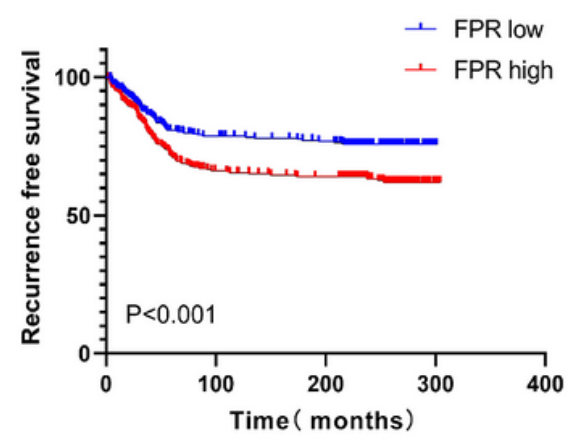

\section{Figure 2}

Kaplan-Meier analysis of preoperative FPR level in 607 patients with esophageal squamous cell carcinoma.FPR was significantly associated with overall survival(a) and recurrence-free survival(b). 
Figure 3

a

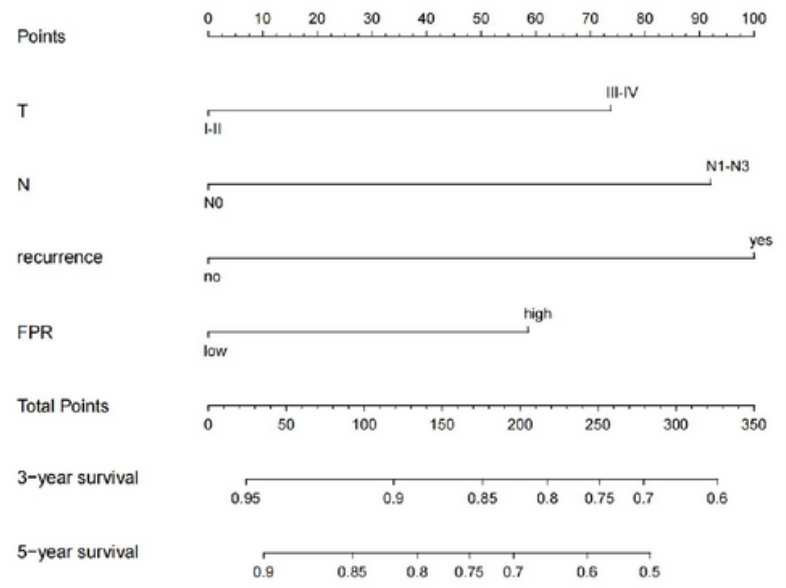

b

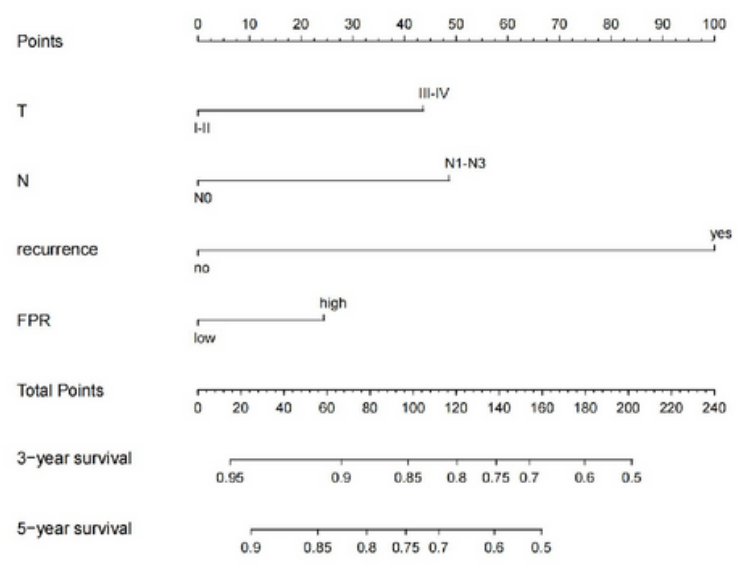

\section{Figure 3}

Nomograms for predicting risk of 3-y and 5-y overall survival (a) and recurrence-free survival (b) in surgical patients with esophageal squamous cell carcinoma.The units of length and width are centimeters $(\mathrm{cm})$. 
Figure 4

a

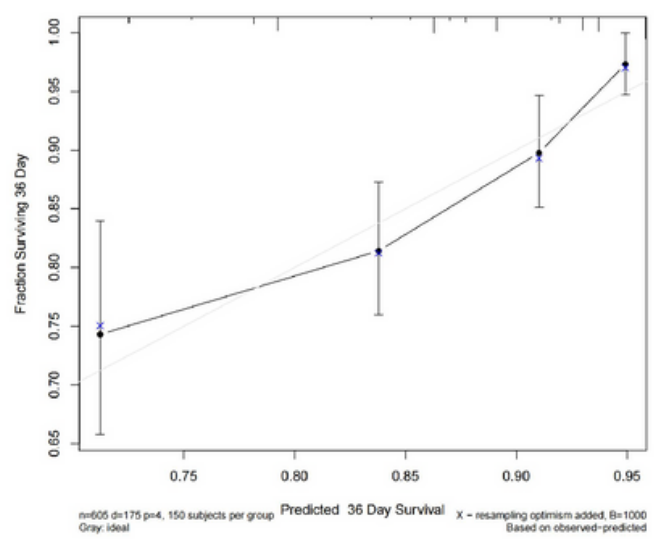

C

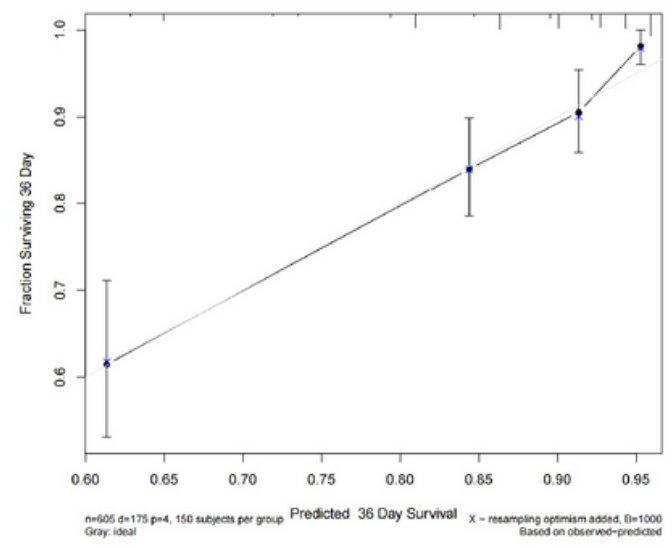

b

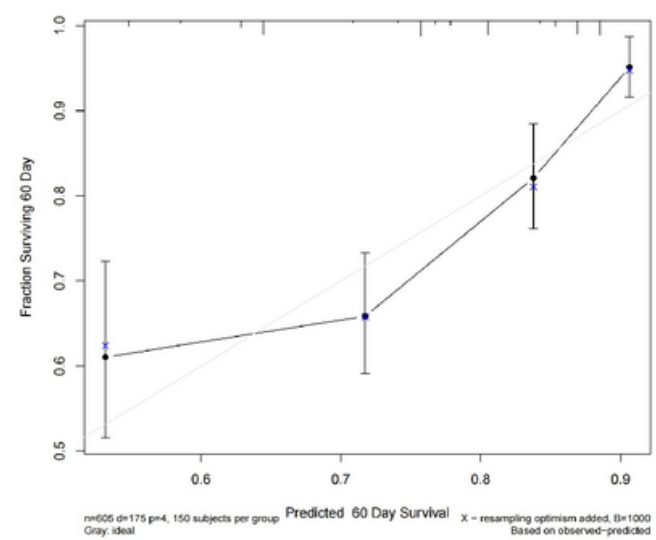

d

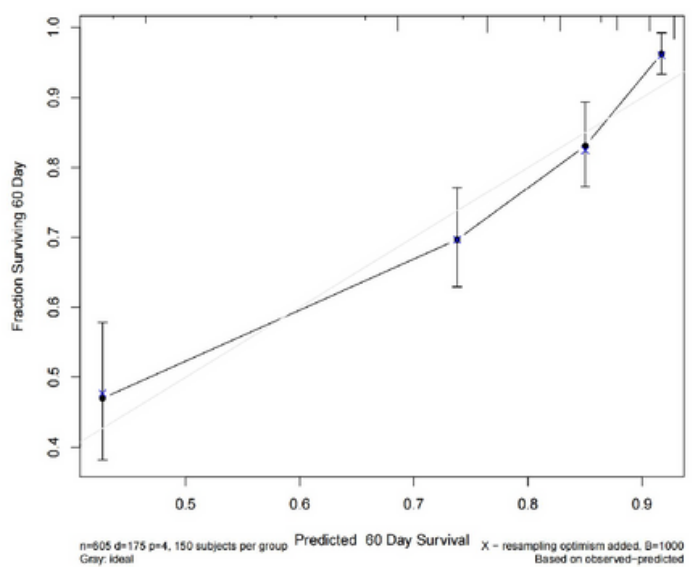

\section{Figure 4}

Calibration curve of nomograms. The predicted 3- and 5-year $(a, b)$ overall survival and recurrence-free survival. (c, d) were well correlated with the actual survival probabilities.

\section{Supplementary Files}

This is a list of supplementary files associated with this preprint. Click to download.

- FPR.tablepptx.pdf 